\title{
Dengue serology-role of NS1 antigen: evaluation and future prospect
}

\author{
Kumar $\mathbf{S}^{1}$, Sandhu $\mathbf{R}^{2}$, Sayal $\mathbf{P}^{3}$, Dahiya $\mathbf{S}^{4}$, Budhani $\mathrm{D}^{5}$ \\ ${ }^{1}$ Dr. Surinder Kumar, Head of Department, ${ }^{2}$ Dr. Raminder Sandhu, Assistant Professor, ${ }^{3}$ Dr. Pallavi Sayal \\ Demonstrator, ${ }^{4}$ Dr. Shalley Dahiya, Medical Officer, ${ }^{5}$ Diksha Budhani, Demonstrator, all authors are attached with \\ Department of Microbiology, BPS, GMC(W), Khanpur Kalan, Sonepat, Haryana, India.
}

Address for Correspondence: Dr. Pallavi Sayal, Demonstrator, Department of Microbiology, BPS, GMC(W), Khanpur Kalan, Sonepat, Haryana, Email: petalz03@gmail.com

\begin{abstract}
Introduction: According to the estimates, nearly $70 \%$ of the disease burden occurs in Asia and is on constant rise. Dengue virus infection has emerged as a major public health concern across the globe in terms of mortality, morbidity and public health cost. Aims \& Objectives: This study was undertaken to evaluate the significance of NS1 Ag detection in dengue IgM negative sera as marker for early detection and to compare the rapid detection test kits (RDT's) with Enzyme linked immunosorbent assay (ELISA). Material \& Method: A total of 5283 samples from, were screened for dengue serology by Immunochromatography based lateral flow assay for presence of IgM and IgG anti Dengue antibody and NS 1 Ag. Results of 169 samples were further confirmed using ELISA technique for NS1 Ag in an attempt to compare RDT's with ELISA. Results: Out of 5283 samples screened, 146/5283 (2.76\%) tested positive for dengue antibody, out of which 85/146 (58.21\%) were positive for DEN Ig M and 61/146 (41.78\%) showed presence of both IgG and IgM. Out of 5137 seronegative samples, 1930 (37.57\%) were positive for DEN NS1 Ag. Overall, sensitivity of RDT was $100.00 \%$, specificity-90.16\%, PPV- 94.73\%, NPV-100.00\% and Efficiency of test-96.44\%. Conclusion: NS1 Ag assay holds promise as useful tool in early diagnosis for detecting dengue infection during acute phase of infection when IgM antibodies are not formed to the detectable levels. Also, accurate and affordable diagnostic tests are a crucial component of combating this debilitating mosquito-borne infection.
\end{abstract}

Key words- Dengue, NS 1 Antigen and Immunochromatographic test (ICT).

\section{Introduction}

Dengue is an arthropod-borne flavivirus that comprises four distinct serotypes (DEN-1, DEN-2, DEN-3 and DEN-4) that constitute an antigenic complex of the genus flavivirus, family Flaviviridae. Infection by one serotype induces life-long immunity against reinfection by the same serotype, but only transient and partial protection against infection with the other serotypes $[1,2]$.

Dengue virus infection can result in clinical manifestations that range from asymptomatic infection to dengue fever (DF) and its severe form termed dengue hemorrhagic fever/dengue shock syndrome (DHF/ DSS). As no protective vaccine or specific treatments are available for dengue, early and accurate laboratory

Manuscript received $4^{\text {th }}$ July 2016

Reviewed: $15^{\text {th }}$ July 2016

Author Corrected: $26^{\text {th }}$ July 2016

Accepted for Publication $9^{\text {th }}$ August 2016 diagnosis is essential for the effective surveillance and control of disease outbreaks [3]. WHO guidelines for diagnosis of dengue relies on virus culture,viral RNA detection, viral antigen detection in tissues by immunechemistry in patients or rising titers of IgG antibody in convalescent patients. But, in resource constrained peripheral health care facilities these tests are remotely available and may compromise with the management of the patients $[4,5]$.

Thus, there is a need for specific, inexpensive dengue diagnostic tests that can be used for clinical management, surveillance and outbreak investigations and would permit early intervention to treat patients and prevent or control epidemics. Only few studies are available that have reported the role of rapid tests used for detection of soluble DENV NS1 Ag in early DENV infection [6-9]. 
Therefore, we undertook this study in an attempt to emphasis significance of detecting DENV NS1 Ag in dengue IgM negative sera, for early detection of infection without the requirement of paired sera. Early diagnosis of DENV infection can improve clinical outcomes by ensuring close follow-up, initiating appropriate supportive therapies and raising awareness to the potential of hemorrhage or shock. Number of Rapid detection tests (RDT's) and Enzyme linked immunosorbent assay (ELISA) based testing formats are available targeting DENV NS $1 \mathrm{Ag}$. Here, we evaluated RDT's in suspected dengue cases to determine their effectiveness for early diagnosis.

\section{Material \& Method}

This retrospective study was conducted in Department of Microbiology in a tertiary care center during an outbreak of Dengue from October 2015 to November 2015. A total of 5283 serum samples from patients identified as probable cases by clinical suspicion (any acute febrile illness with one of the following symptoms: myalgia, headache, retro-orbital pain, bleeding, altered sensorium, shock or low platelet count) attending various outdoor, causality services and indoor patients were received and registered in the study. Informed consent was obtained and a detailed clinical history, physical examination and baseline investigations were undertaken.

Five milliliter of blood was collected from all suspected cases, serum was separated and screened for dengue serology by Immunochromatography (ICT) based RDT lateral flow assay (J Mitra \& Co Pvt Ltd, New Delhi) for presence of IgM and IgG anti- Dengue antibody and NS 1Ag. Tests were performed and results were read as per literature provided.

Out of suspected dengue cases screened by NS $1 \mathrm{Ag}$ RDT, 169 were further tested using ELISA technique (Panbio Dengue Early ELISA) for NS1Ag in an attempt to analyze RDT's results with ELISA.

Data Analysis: Available data was analyzed and sensitivity, specificity, positive predictive value (PPV) and negative predictive value (NPV) were calculated based on confirmed dengue samples by ELISA using the following formulas, where $a$ is the number of true positives, $b$ is the number of false positives, $c$ is the number of false negatives, and $d$ is the number of true negatives: $\%$ sensitivity $=a /(a+c) \mathrm{X} 100 ; \%$ specificity $=d /(b+d) \mathrm{X} 100$; efficiency $=(a+d) /(a+b+c+d) \mathrm{X}$ $100 ; \% \mathrm{PPV}=a /(a+b) \mathrm{X} 100$, and $\% \mathrm{NPV}=d /(c+d) \mathrm{X}$ 100.

\section{Results}

Out of 5283 serum samples from suspected cases during study period, 146/5283 (2.76\%) tested positive for dengue antibody, out of which 85/146 (58.21\%) were positive for DEN Ig M antibody and 61/146 (41.78\%) showed presence of both IgG and IgM (Table 1). Among 5137 seronegative samples, it was observed 1930 (37.57\%) were positive for presence of DEN NS1 Ag and 3207 (62.42\%) were negative for the same.

Table- 1: Results of sample screened for Dengue serology.

\begin{tabular}{|c|c|c|c|c|}
\hline $\begin{array}{c}\text { Total sample } \\
\text { screened }\end{array}$ & NS1 Ag positive & Antibody positive & DEN Ig M Positive & $\begin{array}{c}\text { DEN Ig G + Ig M } \\
\text { Positive }\end{array}$ \\
\hline 5283 & 1930 & 146 & 85 & 61 \\
\hline
\end{tabular}

Among suspected dengue cases screened for NS 1Ag by RDT, 169 were further confirmed by ELISA. Out of the 169, 114 were positive for NS1 Ag RDT by ICT. Out of 114 positive cases, 108 were concordant with the ELISA result and 06 cases were discordant (Table 2).

Table- 2: Comparison of ELISA and RDT for NS1 antigen.

\begin{tabular}{|c|c|c|c|}
\hline & NS 1 ELISA positive & NS 1 ELISA Negative & Total \\
\hline NS1 RDT Positive & 108 & 06 & 114 \\
\hline NS 1 RDT Negative & 0 & 55 & 169 \\
\hline
\end{tabular}

The statistical values obtained were as follows: sensitivity $100.00 \%$, specificity $90.16 \%$, PPV94.73\%, NPV $100.00 \%$, and Efficiency of test $96.44 \%$. 


\section{Discussion}

Laboratory confirmation of dengue infection usually relies on isolation of the virus in cell culture, the identification of viral nucleic acid or antigens, or the detection of virus specific antibodies [10]. Direct virus detection could potentially be used for early, definitive and serotype specific identification of dengue infections during the acute phase of the disease. Live virus or virus components (RNA or antigens) can be detected in serum, plasma, whole blood and infected tissues from 0-7 days following the onset of symptoms, which corresponds roughly to the duration of fever. Direct virus detection procedures are not routinely performed by laboratories, and few commercial kits that have been independently validated are available to aid in this area of dengue diagnosis. Serological tests are more commonly used to diagnose dengue infections because of their ease of use compared to techniques such as cell culture or RNA detection [11].

Results obtained were analyzed and our study showed high prevalence 1930/5137 (37.57\%) of DEN NS1 Ag in DEN IgM seronegative samples, suggesting restricted scope of detecting IgM antibodies as a routine procedure (Figure 1). Our results are in agreement with other investigators as shown in Table 3 who have also reported high sensitivity of DEN NS1 Ag detection during acute phase of infection.

\section{Table 3- NS1 Antigen detection as reported in other studies}

\begin{tabular}{|l|c|c|}
\hline Year & Authors & NS 1 Ag detection \\
\hline 2006 & Dussart et al [7] & $212 / 243(87.20 \%)$ \\
\hline 2007 & Kumarswamy et al [8] & $199 / 213(93.42 \%)$ \\
\hline 2010 & Datta et al [9] & $71.42 \%$ \\
\hline 2012 & Sheemar et al [12] & $59.30 \%$ \\
\hline
\end{tabular}

The dengue serological assays however become more challenging because dengue antibodies are cross reactive with other flavivirus such as West Nile virus (WNV), St. Louis encephalitis virus (SLE), Japanese encephalitis virus (JEV), and yellow fever virus (YFV). In addition, IgM antibody response varies considerably among the individuals due to host humoral immune response or depending on whether a primary vs a secondary infection. [11,13]. Thus, more recently, DENV NS $1 \mathrm{Ag}$ have been reported as being a promising tool for diagnosing acute dengue infections [14-21].

NS1 is a highly conserved nonstructural glycoprotein secreted by virus infected cells during the acute phase of dengue, and it is essential for virus viability [20]. NS1 Ag circulates uniformly in all serotypes of DENV and it circulates at high level during the first few days of illness [6]. NS1 Ag levels varies from $0.04 \mu \mathrm{g} / \mathrm{ml}$ to $2 \mu \mathrm{g} / \mathrm{ml}$ in acute-phase serum samples, to only $0.04 \mu \mathrm{g} / \mathrm{ml}$ or even less in convalescent phase serum [20]. This is the reason for its higher detection rate in acute phase sera. Detection of specific IgM by MAC-ELISA is still used as the diagnostic technique for acute infection; its disadvantage being delayed appearance of antibodies from 5-10 days after the onset of illness in case of primary dengue virus infection and 4-5 days after the onset of illness in secondary infections [9].

The optimal window for diagnosing a dengue infection is roughly from the onset of fever to 10 days post-infection; however, as not all patients are diagnosed within this period, an ideal diagnostic test should be sensitive regardless of the stage of infection [10]. High positivity of DEN NS1 Ag in the seronegative samples for IgM antibodies against DENV indicates the high potential of improving dengue diagnosis by use of NS1 Ag detection assays. Detection of DEN NS1 Ag by RDT's have many advantages including rapidity, convenience and cost-effectiveness. Circulating NS1 has been shown to be detectable from the first day to the early convalescent phase after onset of disease. Further, monoclonal antibody (MAb)-based serotypes specific NS1 assays can be used to differentiate between flaviviruses [17].

Present study evaluated RDT and Elisa testing formats and we have got a sensitivity and specificity of $100.00 \%$, and $90.16 \%$ respectively in detecting NS1 Ag by RDT. Though many studies [15, 22-25] have shown variable sensitivity (65 to $95 \%$ ) and specificity (95 to 100\%) of ICT based RDTs compared with ELISA. This may be due to reason that the ICT may be from different manufactures with different specificities and sensitivities. As rapid ICT kits for the detection of dengue serology have been developed by a number of commercial manufacturers and have found wide application because these require no specialized equipment or training, and results are available within 10 to 30 minutes, making them ideal for low-technology environments. However, these too have limitations like subjective variation in reading and 
substandard performance in acute cases. This demands for large scale evaluation of these kits by authorized agencies. Thus here we emphasized that there is urgent need for validation of RDT's at national level to circumvent the ambiguity of accurate test results in terms of sensitivity and specificity. Limitations of this study is lack of correlation between timing of samples in relation to duration of fever as this study was done using retrospective data. Study results would have been even more informative, had it been done prospectively using a panel of well-characterized samples with proper duration of fever and primary as well as secondary infection. As duration of fever is important in serological diagnosis of DENV infection and it might be one of the reasons for false negative results for dengue when tested for anti IgM antibodies.

\section{Conclusion}

For effective prevention and control of dengue epidemic requires an active laboratory based disease surveillance programme that can provide early warning of impending epidemic transmission. Therefore, NS $1 \mathrm{Ag}$ detection is an effective tool for early diagnosis of DEN infection as compared to Ig M antibody detection. Also our study reinforces the findings of other authors suggesting inclusion of rapid tests in diagnosis and surveillance after proper validation by national agencies for detection of DEN NS1 Ag along with antibody assays. In view of the high mortality rate and to reduce the disease burden, detection of NS $1 \mathrm{Ag}$ and antibody dual assay will help to bridge the gap pointed by initial period of viremia and delayed immune response. Thus, it is imperative to have a rapid and sensitive laboratory assay for early detection of the disease. More work is required to validate accurate and affordable diagnostic rapid card tests as a crucial component for combating this debilitating mosquito borne infection.

Funding: Nil, Conflict of interest: None initiated, Permission from IRB: Yes

\section{References}

1. Halstead SB. Pathogenesis of dengue: challenges to molecular biology. Science.1988 Jan;239(4839):47681.doi:10.1126/science.3277268.

2. Kurane I. Dengue hemorrhagic fever with special emphasis on immunopathogenesis. Comp Immunol Microbiol Infect Dis.2007 Sept 30;(5-6):329-34. doi: 10.1016/j.cimid.2007.05.010.

3. Guzman MG, Kourí, G. Dengue: an update. Lancet Infect Dis.2002 Jan;2(1):33-42. doi:http://dx.doi.org/10. 1016/S1473-3099(01)00171-2.

4. WHO: Dengue: guidelines for diagnosis, treatment, prevention and control, new edition, a joint publication of the World Health Organization (WHO) and the Special Programme for Research and Training in Tropical Diseases (TDR).2009:91-106.
5. Kalyani D, Bai MM. Evaluation of immunechromatographic test in early serological diagnosis of dengue fever. Indian J Pathol Microbiol. 2012 OctDec;55(4):610-1. doi: 10.4103/ 0377-4929.107863.

6. Bessoff K, Delorey M, Sun W, Hunsperger E. Comparison of two commercially available dengue virus (DENV) NS1 capture enzyme-linked immunosorbent assays using a single clinical sample for diagnosis of acute DENV infection. Clin Vaccine Immunol. 2008 Oct 15(10):1513-8. doi:10.1128/ CVI.00140-08.

7. Dussart P, Labeau B, Lagathu G, Louis P, Nunes MR, Rodrigues SG et al. Evaluation of an enzyme immunoassay for detection of dengue virus NS1 antigen in human serum. Clin Vaccine Immunol. 2006 Nov 11;13:1185-9. Doi:10.1128/CVI.00229-06.

8. Kumarasamy V, Wahab AH, Chua SK, Hassan Z, Chem YK, Mohamad $M$ et al. Evaluation of a commercial dengue NS1 antigen-capture ELISA for laboratory diagnosis of acute dengue virus infection. J Virol Methods. 2007 Mar;140(1-2):75-9. Doi:10.1016/j. jviromet.2006.11.001.

9. Datta S, Wattal C. Dengue NS1 antigen detection: A useful tool in early diagnosis of dengue virus infection. Indian J Med Microbiol 2010;28(2):107-10. Doi:10. 4103/0255-0857.62484.

10. Guzman, M G. \& Kouri, G. Dengue diagnosis, advances and challenges. Int J Infect Dis.2004 Mar; 8(2): 69-80. Doi: http: //dx. doi. org/10.1016/j. ijid. 2003.03.003.

11. Vaughn, DW, Green S, Kalayanarooj S, Innis BL, Nimmannitya $S$, Suntayakorn $S$ et al. Dengue viremia titer, antibody response pattern and virus serotype correlate with disease severity. J Infect Dis.2000 Jan;181(1): 2-9. Doi:10.1086/315215.

12. Sheemar S, Mahajan G, Chopra S, Kaur J .Nonstructural protein 1 antigen capture kit as an early 
dengue diagnostic tool. J Med Soc.2012 Dec;26(3):1545. Doi:10.4103/0972-4958.113223.

13. Kao CL, King CC, Chao DY, Wu HL, Chang GJ. Laboratory diagnosis of dengue virus infection: current and future perspectives in clinical diagnosis and public health. J Microbiol Immunol Infect. 2005 Feb;38(1):516. PMID-15692621.

14. Teles FR, Prazeres DM, Lima-Filho JL.Trends in dengue diagnosis. Rev Med Virol. 2005 SeptOct;15(5):287-302. Doi:10.1002/rmv.461.

15. Wang SM, Sekaran SD. Evaluation of a commercial SD dengue virus NS1mantigen capture enzyme-linked immunosorbent assay kit for early diagnosis of dengue virus infection. J Clin Microbiol. 2010 Aug;48(8):27937. Doi:10.1128/JCM.02142-09.

16. Alcon S, Talarmin A, Debruyne M, Falconar A, Deubel V, Flamand M.Enzyme-linked immunosorbent assay specific to Dengue virus type 1 nonstructural protein NS1 reveals circulation of the antigen in the blood during the acute phase of disease in patients experiencing primary or secondary infections. J Clin Microbiol. 2002 Feb;40(2):376-381. Doi:10.1128/JCM. 40.2.376-381.2002.

17. Xu H, Di B, Pan YX, Qiu LW, Wang YD, Hao W et al.Serotype 1-specific monoclonal antibody-based antigen capture immunoassay for detection of circulating nonstructural protein NS1: Implications for early diagnosis and serotyping of dengue virus infections. J Clin Microbiol. 2006 Aug;44(8):2872-8. Doi:10.1128/JCM.00777-06.

18. Hsieh CJ, Chen MJ. The commercial dengue NS1 antigen-capture ELISA may be superior to IgM detection, virus isolation and RT-PCR for rapid laboratory diagnosis of acute dengue infection based on a single serum sample. J ClinVirol. 2009 Jan;44(1):102. DOI:10.1016/j.jcv.2008.10.003

19. Qiu LW, Di B, Wen K, Wang XS, Liang WH, Wang YD et al. Development of an antigen capture immunoassay based on monoclonal antibodies specific for dengue virus serotype 2 nonstructural protein 1 for early and rapid identification of dengue virus serotype 2 infections. Clin Vaccine Immunol. 2009 Jan;16(1):8895. Doi:10.1128/CVI.00212-08.

20. Hang VT, Nguyet NM, Trung DT, Tricou V, Yoksan S, Dung NM et al. Diagnostic accuracy of NS1 ELISA and lateral flow rapid tests for dengue sensitivity, specificity and relationship to viraemia and antibody responses. PLoSNegl Trop Dis 2009,3:e360. Doi:10.1371/journal.pntd.0000360.

21. Huhtamo E, Hasu E, Uzcategui NY, Erra E, Nikkari S, Kantele A, Vapalahti O, Piiparinen H: Early diagnosis of dengue in travelers: comparison of a novel real-time RT-PCR, NS1 antigen detection and serology. J ClinVirol. 2010 Jan;47(1):49-53. Doi:10.1016/j. jcv.2009.11.001

22. Lam SK, Devine PL. Evaluation of capture ELISA and rapid immunochromatographic test for determination of $\operatorname{IgM}$ and $\operatorname{IgG}$ antibodies produced during dengue infection. ClinDiagnVirol 1998 May; 10(1):75-81. PMID:9646004.

23. Vianney T, Hang TT Vu, Nhu VN Quynh, Chau VV Nguyen, Hien T Tran, Jeremy Farrar et.al. Comparison of two dengue NS1 rapid tests for sensitivity, specificity and relationship to viraemia and antibody responses. BMC Infect Dis 2010;10:142. Doi: 10.1186/1471-233410-142

24. Pal S, Dauner AL, Mitra I, Forshey BM, Garcia P, Morrison AC et al. Evaluation of Dengue NS1 antigen rapid tests and ELISA kits using Clinical samples. PLoS ONE. 2014 Nov;9(11);1-8. Doi:http://dx.doi.org/10. 1371/journal.pone.0113411

25. Sahu SK, Ghosh SK, Beheral SK,Mohanty DP, Thakur S. Use of rapid test kit and ELISA for detection of NS1 Antigen and Ig M antibody in suspected dengue fever. Int J Microbiol parasite. 2015 Feb 16;1(1);1-3.

\section{How to cite this article?}

Kumar S, Sandhu R, Sayal P, Dahiya S, Budhani D. Dengue serology-role of NS1 antigen: evaluation and future prospect. Int J Med Res Rev 2016;4(8):1335-1339.doi:10.17511/ijmrr.2016.i08.10. 